\title{
CLASSIFICATION OF AMYOTROPHIC LATERAL SCLEROSIS AND HEALTHY ELECTROMYOGRAPHY SIGNALS BASED ON TRANSFER LEARNING
}

\author{
Abdulkadir ŞENGÜR ${ }^{* 1}$, Ümit BUDAK ${ }^{2}$, Yaman AKBULUT ${ }^{3}$ \\ ${ }^{* 1}$ Electrical and Electronics Engineering Dept., Technology Faculty, Firat University, Elazig, Turkey \\ ${ }^{2}$ Electrical and Electronics Eng. Dept., Engineering Faculty, Bitlis Eren University, Bitlis, Turkey \\ ${ }^{3}$ Informatics Dept., Firat University, Elazig, Turkey \\ *Corresponding author; E-mail: ksengur@gmail.com
}

Received: 16 December 2018; Accepted: 29 December 2018

\begin{abstract}
This paper investigates the usage of transfer learning in amyotrophic lateral sclerosis (ALS) disease detection. ALS is a dangerous disease which affects the nerve cells in brain and spinal cord. Electromyogram (EMG) is an important measure for analysing of the electrical level of the muscles. EMG based early ALS disease detection system helps the physicians and patients. The proposed work uses EMG signals in discrimination of the ALS and healthy persons. The EMG signals are initially segmented with a overlapped window and each segment is converted to the spectrogram images. The obtained spectrogram images are resized and fed into the pre-trained convolutional neural networks model. The pre-trained model is fine-tuned with the problem at hand. The R002 dataset which is obtained from www.emglab.net is used during the experimental works. Accuracy, sensitivity and specificity measures are used to evaluate the obtained achievement. According to these measures, 97.70\% accuracy, 97.97\% sensitivity, and 97.29\% specificity values are recorded. We further compare the obtained results with some of the existing results that were obtained on the same dataset. The comparisons show that proposed method is outperformed.
\end{abstract}

Key words: EMG signals, ALS disease, Transfer learning, Convolutional neural networks, Pre-trained models.

\section{Introduction}

Electromyography is an essential tool for pyhsicians who monitor and evaluate the electrical activity of the muscles [1]. Electromyogram (EMG) is an electrical signal which is useful in various fields such as exercise physiology and medical sciences [2]. EMG signals have been used in detection of the amyotrophic lateral sclerosis (ALS) disease [3]. ALS is a dangerous disease which affects the nerve cells in brain and spinal cord. Thus, the patients can not control his/her muscles and cramping of muscles, nasal speech and difficulty in chewing or swallowing can be seen frequently. 
Computer aided diagnosis (CAD) systems help physicians in many fields. EMG based CAD systems are also in demand for detection of various disease such as ALS. Doulah et al. [4] classified EMG signals into normal and ALS classes based on features that were obtained with mel-frequency cepstral coefficient. K-nearest neighbor (K-NN) classifier was adopted in the classification phase of the proposed work. The proposed method achieved 92.50\% accuracy. Mishra et al. [5] used empirical mode decomposition (EMD) for classification of the EMG signals. Six different features were considered which were obtained from the EMD of the EMG signals. Least-Square Support Vector Machine (LSSVM) technique was used in the classifcation stage of the work. Authors reported $95.00 \%$ classification accuracy for their work. Sengur et al. [6] used time-frequency images and convolutional neural networks (CNN) for differenting of the EMG signals into ALS and normal classes. An end-to-end CNN model was trained with spectrogram images of the randomly sampled EMG signals. The achievement of the proposed method was 96.69\%. Fattah et al. [7] used discrete wavelet transform (DWT) for EMG signal analysis. The authors used frame of the EMG signals instead of the whole EMG signal for di scriminating of the ALS diseases. Several statistical measures such as maximum and average values on the DWT coeffients were used as features. The k-NN classifier was used for classification and $100 \%$ classification accuracy was presented by the authors. Pal et al. [8] used various features such as spectrogram, rootmean-square, entropy, and kurtosis for detection of the ALS from EMG signals. Merlo et al. [9] proposed a fast and reliable method for EMG signal analysis. The authors used wavelet transform for feature extraction. The on and off timing of skeletal muscles at the time of movement was investigated. Sengur et al. [10] proposed time-frequency analysis methods for EMG signal classification. Authors used spectrogram, WT, and Wigner Ville distribution methods. CNN and reinforcement sample learning strategy were then employed to classify these features.

In this paper, a deep learning architecture is used for EMG signal classification. Specifically, the transfer learning (TL) is considered due to its simplicity. TL is the current trend in deep learning, where knowledge of a pre-trained network is shared or transfer to another network [11]. In TL, first, a CNN model is trained by a larger dataset then this pre-trained model is again trained by a smaller dataset to obtain fine tuning for improving the predictive performance of CNN model. The AlexNet model is used as pre-trained CNN model [12]. The color images, that are fed to TL model, are obtained via spectrogram representation of the EMG signals. An overlapping window is used to sample the EMG signals for producing the input images. The input images are then resized to $227 \times 227$. The TL is trained with by using the stochastic gradient descent with momentum. The R002 dataset which is obtained from www.emglab.net [13], is used during the experimental works. Accuracy, sensitivity and specificity measures are used to evaluate the obtained achievement. According to these measures, $97.70 \%$ accuracy, $97.97 \%$ sensitivity, and $97.29 \%$ specificity values are recorded. We further compare the obtained results with some of the existing results that were obtained on the same dataset. The comparisons show that the proposed method is outperformed.

\section{Material and Methods}

\subsection{Convolutional Neural Networks}

With the recent developments in deep learning, applications with convolutional neural networks (CNNs) become a shining title for researchers. Generally speaking, CNNs were introduced as a category of feed-forward neural networks $[12,14]$. CNNs are compact structures where feature extraction and 
classification is handled in one structure. CNNs consist of multiple layers such as convolution, pooling, and fully connected layers. Convolution layer contains a bunch of filters which are convolved across the width and height of the input volume in the forward pass. These filters' coefficients are assigned randomly in the beginning and then filter coefficients are adapted during the training procedure. Pooling layer aims to create a non-linear down-sampling. In other words, pooling partitions the input volume into a set of non-overlapping rectangle sub-regions and for each sub-region, the considered operation is applied for obtaining the output. Fully connected layer is responsible for classification procedure. Neurons in fully connected layer have full connections to all activations in the previous layer.

\subsection{Transfer Learning}

Using a pre-trained CNN model either for feature extraction or fine-tuning is called transfer learning [15]. Transfer learning uses the knowledge of a pre-trained network, which was trained with millions of labeled images, for a new problem where the number of input image is limited. In the application of the transfer learning, most of the layers of the pre-trained CNN model are saved except the last three layers namely fully connected layer, softmax layer, and classification output layer.

\section{Proposed Method}

The illustration of the proposed method is given in Fig. 1. As seen in Fig. 1, the input EMG signals are initially sampled with an overlapped window for segmentation of the EMG signals. The overlapped window is used for acquiring more EMG segments. In other words, data augmentation is handled with overlapped windowing procedure.

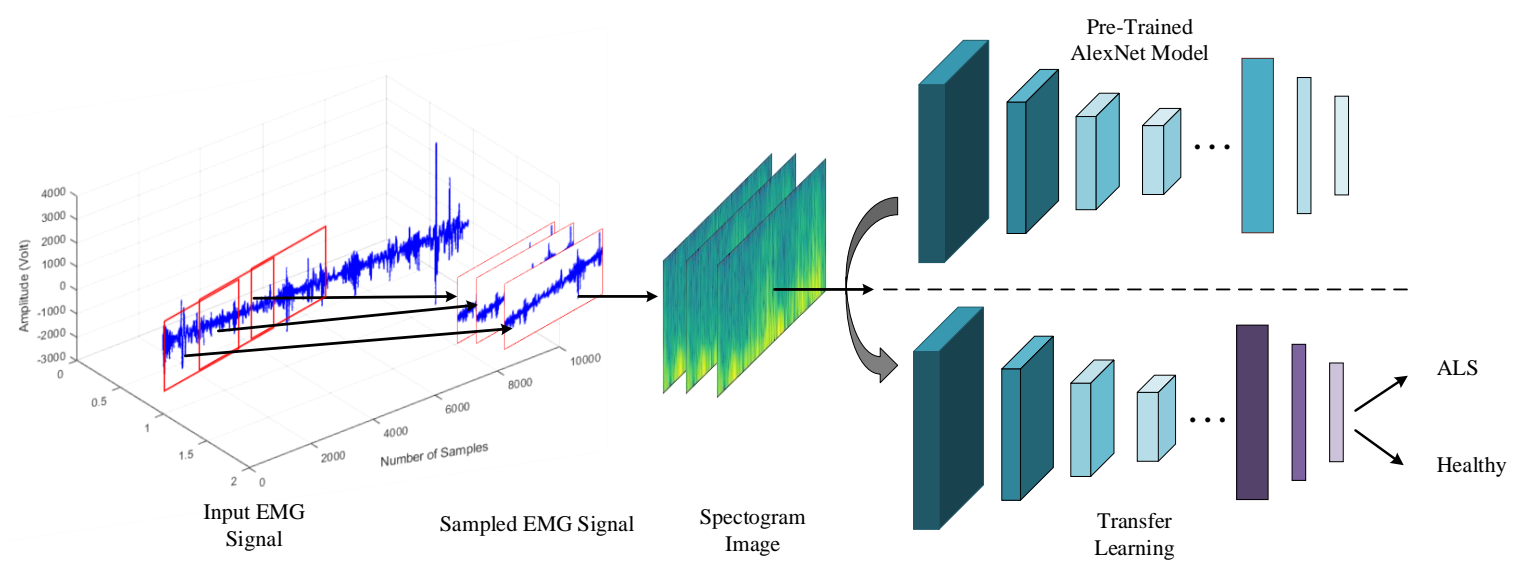

Figure 1. The proposed transfer learning methodology for EMG signal classification

The original EMG signals have around 260,000 samples and the length of the sampling window is chosen as 10,000 samples. The sampled EMG signals are then converted into the color images by applying the time-frequency (T-F) transformation. The short time Fourier transform is considered for T-F image transformation. The constructed T-F images are resized to $227 \times 227$. The AlexNet model is considered as a pre-trained $\mathrm{CNN}$ model which was trained on ImageNet challenge [12]. AlexNet is known to be the first deep CNN model that was introduced by Krizhevsky et al. [12]. AlexNet model is totally comprised of 25 layers where 5 of them contain learnable weights and last three layers cover fully connected layers. In AlexNet architecture, rectified linear units, normalization, and max-pooling 
layers come after the convolutional layers and convolutional layers use varying kernel sizes. The last three layers of the AlexNet model are discharged because that layers were configured for 1,000 classes ImageNet challenge. These three layers are fine-tuned for the EMG signal classification problem where the number of classes is 2 .

\section{Experimental Works and Results}

The efficiency of the transfer learning is showed on the dataset that was collected by the University of Copenhagen in 2001 [16]. Six males and four females were used for collecting of the healthy EMG signals and four males and four females ALS patients were used for ALS diseased EMG signal collection. The healthy group has an age interval from 21 to 37 years old and ALS group covers the age interval from 35 to 67 years old. A standard needle electrode was used for the signal acquisition. Sampling rate of EMG signals was almost $24 \mathrm{kHz}$ and digitized by A/D convertor of 16-bit resolution. Recorded signals were filtered at $2 \mathrm{~Hz}$ and $10 \mathrm{kHz}$ by high- and low-pass filters, respectively.

The dataset contains totally 202 EMG signals of which 89 of them were collected from ALS patients and 133 of them were collected from healthy persons. An overlapping window is used to sample the EMG signals for producing the input spectrogram images as shown in Fig. 1. In spectrogram creation of the EMG signals, we used Hamming window of with $48 \mathrm{~ms}$ and overlap $32 \mathrm{~ms}$ and the number of the FFT was chosen as 512. The parameters for the spectrogram were determined heuristically during the experimental works. After these sampling procedures, 8,842 spectrogram images were constructed for ALS class and 13,420 spectrogram images were recorded for healthy class. Fig. 2 shows sample spectrogram images from both ALS and healthy classes. While the first row of the Fig. 2 shows the ALS EMG images, the second row shows the healthy EMG images.
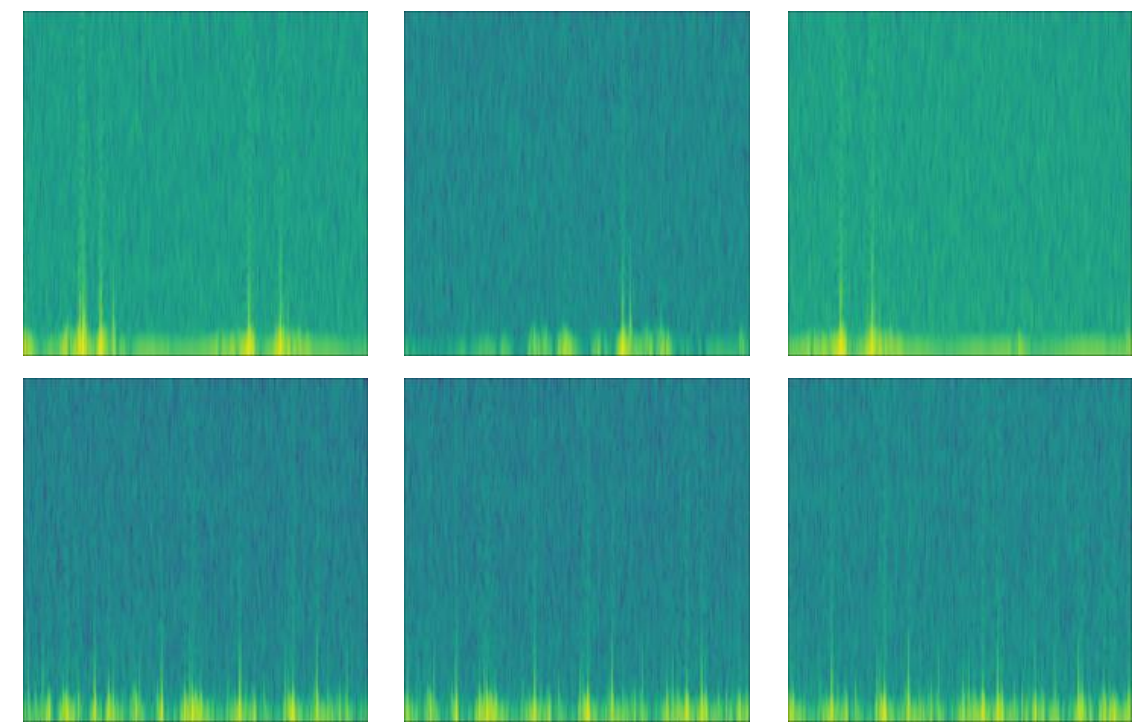

Figure 2. Spectrogram images for the ALS and healthy classes. The first row shows sample images from ALS class and the second row shows sample images from healthy class

For fine-tuning of the CNN model, the mini batch size was chosen as 10 and the initial learning rate was assigned as 0.0001 . The initial learning rate was chosen small enough to slow down learning in the transferred layers. The maximum epoch number was set to 7 and the CNN model was trained by 
stochastic gradient descent with momentum. The training procedure is ended around 6,000 iterations. Fig. 3 shows the training progress of fine-tuned AlexNet model. While the first row shows the deviation of the accuracy against iterations, the second row shows the loss deviation against iterations. It is worth to mentioning that cross validation test was not applied for transfer learning. The $75 \%$ data was used for fine-tuning of the AlexNet model and the rest $25 \%$ was used for testing the fine-tuned CNN model.

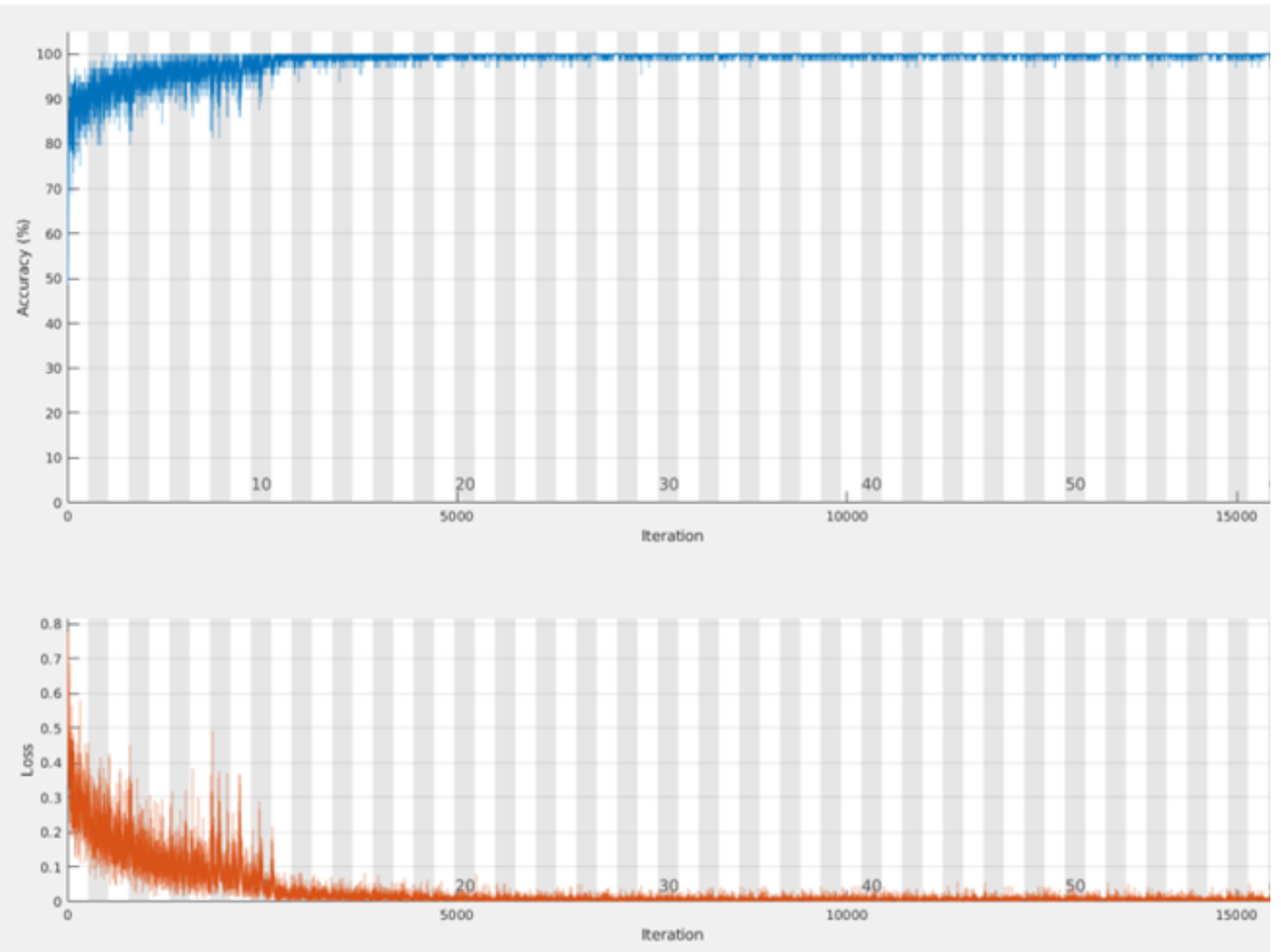

Figure 3. The training progress of fine-tuned AlexNet model

The obtained results were evaluated based on sensitivity, specificity and accuracy criterions respectively as shown in Table 2 . In addition, the confusion matrix is given in Table 1.

Table 1. The confusion matrix

\begin{tabular}{|r|c|c|}
\hline & ALS & Healthy \\
\hline ALS & 2158 & 60 \\
\hline Healthy & 68 & 3287 \\
\hline
\end{tabular}

As seen in Table 2, 97.70\% accuracy, $97.29 \%$ specificity, and $97.97 \%$ sensitivity values were obtained.

Table 2. Performance criteria of proposed method

\begin{tabular}{cccc}
\hline Method & Sensitivity & Specificity & Accuracy \\
\hline Transfer learning & $97.97 \%$ & $97.29 \%$ & $97.70 \%$ \\
\hline
\end{tabular}


Performance comparison of the proposed method with some other methods from literature was given in Table 3. As seen in Table 3, the proposed method outperformed for sensitivity and accuracy evaluation metrics and Sengur et al.'s method produced best specificity value. The proposed method's accuracy was $0.9 \%, 2.7 \%$, and 5.2\% higher than Sengur et al.'s, Mishra et al.'s and Doulah et al.'s methods, respectively.

Table 3. Performance comparison with other methods. The bold case shows the best values

\begin{tabular}{cccc}
\hline Method & Sensitivity & Specificity & Accuracy \\
\hline Doulah et al. [4] & $76.00 \%$ & $98.00 \%$ & $92.50 \%$ \\
Mishra et al. [5] & $93.00 \%$ & $92.50 \%$ & $95.00 \%$ \\
Sengur et al.[6] & $94.80 \%$ & $\mathbf{9 8 . 8 0 \%}$ & $96.80 \%$ \\
Proposed method & $\mathbf{9 7 . 9 7 \%}$ & $97.29 \%$ & $\mathbf{9 7 . 7 0 \%}$ \\
\hline
\end{tabular}

\section{Conclusions}

In this paper, transfer learning is used to detect ALS disease from EMG signals. EMG signals are segmented with an overlapped window and then transformed into the time-frequency images. The spectrogram images were used as input of CNN. Therefore the input images were scaled with $227 \times 227$ dimensions.The pre-trained AlexNet model is used for fine tuning. For fine-tuning of the CNN model, the mini batch size was chosen as 10 and the initial learning rate was assigned as 0.0001 . The initial learning rate was chosen small enough to slow down learning in the transferred layers. The maximum epoch number was set to 7 and the CNN model was trained by stochastic gradient descent with momentum. The obtained classification results are evaluated based on some metrics such as accuracy, sensitivity, and specificity. The evaluation metrics show the efficiency of the transfer learning in detection of the ALS disease. We further compare the obtained results with some of the published results. Comparisons show that the proposed method is outperformed. It is worth to mentioning that the fine-tuning procedure is depended on the number of input data. In addition, the training procedure is time-consuming and necessitates strong GPU devices.

\section{References}

[1] Fuglsang- Frederiksen, A., The utility of interference pattern analysis, Muscle \& Nerve: Official Journal of the American Association of Electrodiagnostic Medicine, 23(1), 2000, pp. 18-36.

[2] Fukuda, T. Y. et al., Root mean square value of the electromyographic signal in the isometric torque of the quadriceps, hamstrings and brachial biceps muscles in female subjects, Journal of Applied Research, 10(1), 2010, pp. 32-39.

[3] Fattah, S. A. et al., Evaluation of different time and frequency domain features of motor neuron and musculoskeletal diseases, Int. J. Comput. Appl., 43(23), 2012, pp. 34-40.

[4] Doulah, A. B. M. S. U., Fattah, S. A., Neuromuscular disease classification based on mel frequency cepstrum of motor unit action potential, In Electrical Engineering and Information \& Communication Technology (ICEEICT), 2014 International Conference on IEEE, pp. 1-4. 
[5] Mishra, V. K. et al., Analysis of ALS and normal EMG signals based on empirical mode decomposition, IET Science, Measurement \& Technology, 10(8), 2016, pp. 963-971.

[6] Sengur, A. et al., DeepEMGNet: an application for efficient discrimination of ALS and normal EMG signals, T. Bvrezina, R. Jabłoński (Eds.), Mechatronics 2017 Recent Technol. Sci. Adv., Springer International Publishing, Cham (2018), pp. 619-625, Doi: 10.1007/978-3-319-65960$2 \_77$.

[7] Fattah, S. A. et al., Identification of motor neuron disease using wavelet domain features extracted from EMG signal, In Circuits and Systems (ISCAS), International Symposium on IEEE, 2013, pp. 1308-1311.

[8] Pal, P. et al., Feature extraction for evaluation of Muscular Atrophy, In Computational Intelligence and Computing Research (ICCIC), International Conference on IEEE, December 2010, pp. 1-4.

[9] Merlo, A. et al., A fast and reliable technique for muscle activity detection from surface EMG signals, IEEE Transactions on Biomedical Engineering, 50(3), 20113, pp. 316-323.

[10] Sengur, A. et al., Classification of amyotrophic lateral sclerosis disease based on convolutional neural network and reinforcement sample learning algorithm, Health information science and systems, 5(1), 2017, 9. Doi: 10.1007/s13755-017-0029-6

[11] Orenstein, E. C., Beijbom, O., Transfer Learning and Deep Feature Extraction for Planktonic Image Data Sets, In Applications of Computer Vision (WACV), Winter Conference on IEEE, March 2017, pp. 1082-1088.

[12] Krizhevsky, A. et al., Imagenet classification with deep convolutional neural networks, In Advances in neural information processing systems, 2012, pp. 1097-1105.

[13] Nikolic, M., Detailed analysis of clinical electromyography signals: EMG decomposition, findings and firing pattern analysis in controls and patients with myopathy and amyotrophic lateral sclerosis, 2001, Doctoral dissertation.

[14] Simonyan, K., Zisserman, A., Very deep convolutional networks for large-scale image recognition, 2014, arXiv preprint arXiv:1409.1556.

[15] Deniz, E., et al., Transfer learning based histopathologic image classification for breast cancer detection, Health information science and systems, 2018, 6(1), 18.

[16] Detailed analysis of clinical electromyography signals EMG decomposi-tion, findings and firing pattern analysis in controls and patients with myopathy and amy-trophic lateral sclerosis. Ph.D. Thesis, Faculty of Health Science, University of Copenhagen. 